DSF-T-95/12

INFN NA-IV-95/12

CPTMB/PT/95-2/

\title{
Consistent anomalies of the induced W gravities $^{\dagger}$
}

\author{
Mario Abud ${ }^{(1)}$, Jean-Pierre Ader ${ }^{(2)}$ and Luigi Cappiello ${ }^{(1)}$ \\ (1) Dipartimento di Scienze Fisiche, Università di Napoli and INFN Sezione di \\ Napoli, Mostra d'Oltremare, Pad. 19, I-80125 Napoli, Italy. \\ (2) Centre de Physique Théorique et de Modélisation, CNRS ${ }^{\dagger \dagger}$, Université de \\ Bordeaux I, \\ 19 rue du Solarium, F-33175 Gradignan, France.
}

\begin{abstract}
The BRST anomaly which may be present in the induced $W_{n}$ gravity quantized on the light-cone is evaluated in the geometrical framework of Zucchini. The cocycles linked by the cohomology of the BRST operator to the anomaly are straightforwardly calculated thanks to the analogy between this formulation and the Yang-Mills theory. We give also a conformally covariant formulation of these quantities including the anomaly, which is valid on arbitrary Riemann surfaces. The example of the $W_{3}$ theory is discussed and a comparison with other candidates for the anomaly available in the literature is presented.
\end{abstract}

$\dagger$ Partially supported by INFN and MURST $60 \%$.

†† Unité Associée au CNRS, U.R.A. 1537. 


\section{Introduction}

In this letter we study the cohomology linked to the consistent anomaly possibly occuring in the induced $W_{n}$ gravities. They are higher spin generalizations of 2-dim gravity whose symmetries are the $W$-algebras, just as the Virasoro algebra appears as the residual symmetry after gauging of 2-dim induced gravity. For recent reviews see [1, 2]. This anomaly and the cocycles linked to it by this cohomology are calculated using Zucchini's formalism [3]. Recently, this author has observed that the generalized Beltrami differentials and projective connections which are dynamical fields appearing in the induced light-cone $W_{n}$ gravity are geometrical objects parametrizing in one-to-one fashion generalized projective structures on a given Riemann surface $\Sigma$. This approach allows us to realize the $W_{n}$ symmetry in terms of an explicit parametrization of a 2-dim Yang-Mills (Y-M) connection. Thus the well-known cohomology of Y-M leads straightforwardly to the formulation of the cohomology of any $W_{n}$-model. After a general presentation of this geometric approach, we give explicit results in the case of $W_{3}$ theory and compare with a previously known candidate for the anomaly [4] in that case.

\section{Zucchini's approach.}

We recall that an atlas of projective coordinates on a Riemann surface $\Sigma$ is a collection of local homomorphisms $Z$ of $\Sigma$ into $C^{1}$ which are glued on overlapping domains by a Moebius transformation. Such an atlas defines a complex structure on $\Sigma$, or equivalently conformal classes of metrics which are related to the reference structure $z$ by the Beltrami coefficient $\mu_{\bar{z}}^{z}$ via Beltrami's equation. This projective structure $A$ is in one-to-one correspondence with the pairs $\left(\mu_{\bar{z}}^{z}, \rho_{z z}\right)$ where $\rho_{z z}$ is the projective connection (the Schwarzian derivative of $Z$ ). The algebra which underlies this framework is the famous Virasoro algebra, corresponding to the $W_{2}$ case.

The approach [3] of the algebra $W_{n}$, where $n$ indicates the highest spin of the generators involved, is based on a straightforward generalization of the notion of projective coordinates. It consists in enlarging the set of coordinates by considering a collection of local maps $\left(Z^{1}, \ldots, Z^{n-1}\right)$ of $\Sigma$ into $C^{n-1}\left(Z^{1} \equiv Z\right)$ t. On overlapping domains $K_{\alpha}, K_{\beta}$ the transition functions of these local maps $Z_{\alpha}^{i}$ define a $S l(n ; \mathbb{C})$-valued 1-cocycle $\Phi_{\alpha \beta}$ on $\Sigma$ which in turn defines a flat $S l(n ; \mathbb{C})$ vector bundle $\Phi$ on $\Sigma$. Such bundle is canonically associated to the generalized projective structure $A$. These new data on the Riemann surface are used to build a basic object, the matrix $\mathrm{W}$, with entries

$$
W_{i}^{r}=\partial^{i}\left(\Delta^{-\frac{1}{n}} Z^{r}\right)
$$

where $\partial_{z} \equiv \partial$ and $\Delta=\operatorname{det}\left\|\partial^{p} Z^{l}\right\|$. One can easily verify that $\operatorname{det} W=1$.

\footnotetext{
${ }^{1}$ We consider only the holomorphic sector of the theory, all results derived below being transposed to the antiholomorphic sector by complex conjugation.
} 
Under a holomorphic coordinate change on $\Sigma$ and a map change of the projective atlas, one has

$$
W_{b \beta}=\Lambda_{b a} W_{a \alpha}\left(\Phi^{-1}\right)_{\alpha \beta}^{t}
$$

where $\Phi_{\alpha \beta}$ is the $S l(n ; \mathbb{C})$ cocycle defined above and $\Lambda_{a b}$ are the transition matrices of the bundle of $(n-1)$-jets of sections of $k^{(1-n) / 2}$, where $k$ is the canonical line bundle with transition functions $k_{b a} \equiv \partial_{b} z_{a}$.

One further define the two matrices

$$
\Omega=\partial W W^{-1} \quad ; \quad \Omega^{*}=\bar{\partial} W W^{-1},
$$

which are traceless and independent of the choice of a chart in the projective atlas. They glue under conformal coordinate changes as follows:

$$
\begin{gathered}
\Omega_{b}=k_{b a}\left(\Lambda_{a b} \Omega_{a} \Lambda_{a b}^{-1}+\partial \Lambda_{a b} \Lambda_{a b}^{-1}\right), \\
\Omega_{b}^{*}=\bar{k}_{b a}\left(\Lambda_{a b} \Omega_{a}^{*} \Lambda_{a b}^{-1}\right) .
\end{gathered}
$$

The Wronskian form of $W$ implies that the elements of $\Omega$ are trivial (zero or one) except $n-1$ elements $\rho_{i} \equiv \Omega_{n-1}^{i}$ which characterize the maps $\left(Z^{1}, \ldots, Z^{n-1}\right)$. By taking suitable combinations of these objects and their derivatives [5, 6] new fields $\left(\tilde{\rho}_{i} ; i=1, . ., n-2 ; i \neq 0\right)$ are constructed which have definite conformal spins $i+2$. Moreover the field $\tilde{\rho}_{n-2}$ is a projective connection transforming as

$$
\tilde{\rho}_{n-2_{b}}=k^{2}\left(\tilde{\rho}_{n-2_{a}}+\frac{n\left(n^{2}-1\right)}{12} S\left(z_{b}, z_{a}\right)\right)
$$

where $S\left(z_{b}, z_{a}\right)$ is the Schwarzian derivative given by

$$
S\left(z_{b}, z_{a}\right)=\partial_{a}^{2} \ln \partial_{a} z_{b}-\frac{1}{2}\left(\partial_{a} \ln \partial_{a} z_{b}\right)^{2}
$$

The matrices $\Omega$ and $\Omega^{*}$ can be considered as the two components of a 2 -dim flat connection since they satisfy the relation

$$
\bar{\partial} \Omega-\partial \Omega^{*}+\left[\Omega, \Omega^{*}\right]=0 .
$$

These flatness conditions first allow us to determine elements of $\Omega^{*}$ in terms of $\Omega$ and of $n-1$ fundamental fields $\mu_{i} \equiv \Omega_{i}^{* n-1}(i=1, . ., n-1)$ which are the generalizations of the usual Beltrami coefficient. They also give the holomorphic conditions obeyed by the $\tilde{\rho}_{i}$. For $n=2$ these conditions reduce to the unique relation

$$
\left(\bar{\partial}-\mu_{0} \partial-2 \partial \mu_{0}\right) \rho_{0}=-\frac{1}{2} \partial^{3} \mu_{0}
$$


which is exactly the anomalous Ward identity of the induced chiral 2-dim gravity, once the field $\rho_{0}$ has been identified with the induced energy-momentum tensor and $\mu_{0}$ (the external source coupled to it) with the Beltrami differential on $\Sigma$.

The relation between 2-dim induced gravity and a 2-dim partially gauge fixed $S l(2 ; \mathbb{R})$ Y-M theory was first found by Polyakov [7]. In ref. [8], the generalization of Polyakov's gauge-fixing on an arbitrary Riemann surface (see also ref. [9]) led to the introduction of $W$ matrices of $S l(2 ; \mathbb{C})$ depending on a single projective coordinate. The construction made in [3] suggests the interpretation of the holomorphic conditions deduced from (77) as the Ward identities of the induced $W_{n}$ gravity, once the correct identifications for the fields $\tilde{\rho}_{i}$ (and the corresponding generalized Beltrami differentials $\tilde{\mu}_{i}$ ) have been made. The association of a zero curvature condition to the formulation of induced $W_{n}$ gravity and its interpretation as an anomaly equation are not new [10, 11]. The essential advantage of this geometrical framework is to define the $W_{n}$ symmetries as gauge transformations of the vector bundle $\Phi$ and to provide a systematic method to derive the corresponding nilpotent BRST algebra, as we now discuss.

\section{BRST symmetry}

The vector bundle $\Phi$ can be considered as a functional of the fields $\left(\tilde{\rho}_{i}, \tilde{\mu}_{i}\right)$; the variations of these fields leaving this bundle invariant are precisely the form of the $W_{n}$ symmetry transformations. They are obtained from infinitesimal variations of the maps $Z$ of $A$ [3]

$$
\delta Z^{r}=\epsilon_{s}^{r} Z^{s}-\epsilon_{s}^{0} Z^{r} Z^{s}
$$

which when written in terms of a ghost matrix field $\gamma$, instead of infinitesimal parameters $\epsilon$, give the corresponding BRST transformations. The matrix $\gamma$ has zero trace and transforms under changes of trivialization of the bundle $\Phi$ as

$$
\gamma_{\beta} \Phi_{\beta \alpha}=\Phi_{\beta \alpha} \gamma_{\alpha}
$$

Geometrically, this means that the matrix function $\gamma$ corresponds to a finite gauge transformation of $\Phi$. This allows to construct [3] with the help of $\gamma$ and $W$ a traceless matrix $C$ (which has a ghost grading one) which is independent of map choices in the projective structure. Moreover, under a conformal coordinate change in the canonical bundle $k$ this ghost matrix transforms as

$$
C_{b}=\Lambda_{b a} C_{a} \Lambda_{b a}^{-1}
$$

Now nilpotency of the law (9) is fulfilled if $s \gamma=\gamma^{2}$ and the BRST transformation of the matrix $W$ is then

$$
s W=C W .
$$


By considering this equation as the definition of $C$, it appears that $C$ is thus not only a function of the ghosts $\gamma$ but also of the variables $Z^{i}$ (in fact the $\rho_{i}$ ). Its explicit form can be deduced from (11) and will not be given here. It can be found in ref.[3].

Moreover

$$
s C=C C .
$$

Obviously this transformation is nilpotent and looks like the well-known BRST transformation of the Faddeev-Popov ghost in Y-M theory. From eq. (12) and the definition (3) of the matrix $\Omega$ one has

$$
s \Omega=\partial C+[C, \Omega] .
$$

Analogously it is straightforward to deduce the BRST transformation of $\Omega^{*}$

$$
s \Omega^{*}=\bar{\partial} C+\left[C, \Omega^{*}\right] .
$$

The parallel with the Y-M formalism leads us to gather together the two matrices $\Omega$ and $\Omega^{*}$ into a 2 -dim gauge connection

$$
\mathcal{A}=\Omega d z+\Omega^{*} d \bar{z}
$$

which transforms as ( $d$ being the usual external derivative)

$$
s \mathcal{A}=-d C+[\mathcal{A}, C] .
$$

The curvature (field strength) $\mathcal{F}=d \mathcal{A}-\mathcal{A A}$, corresponding to the connection $\mathcal{A}$ is in fact zero due to the holomorphy condition (7).

It is well known that the Ward identities for induced $W$ gravity are very similar in structure to the BRST transformations of the projective connection and the currents. For 2-dim gravity (i.e $W_{2}$ ) this is a direct consequence of the analogy between the Beltrami equation $\bar{\partial} Z=\mu_{\bar{z}}^{z} \partial Z$ and the BRST transformation $s Z=$ $c \partial Z$, where $c$ is the ghost introduced by Becchi [12]. Accordingly every function of the projective coordinate $Z$ and of $(\partial, z)$ will exhibit this relation between its BRST transformation and the relation induced by the Beltrami equation. The same sort of relation is present in the $W_{n}$ models and was previously discussed in ref [13]. This is obvious if we compare the transformation law (12) with the definition (3) of the matrix $\Omega^{*}$ considered as the holomorphic condition verified by $W$. In fact the relation (14) can be deduced from the compatibility condition (77) between $\Omega$ and $\Omega^{*}$ by replacing the matrix $\Omega^{*}$ and the partial derivative $\bar{\partial}$ by the ghost matrix $C$ and the BRST operator $s$ respectively.

Thus the matrix $C$ is straightforwardly deduced from the matrix $\Omega^{*}$ by replacing the generalized Beltrami coefficients $\mu_{i}$ by the ghost fields $c_{i}$ and consequently there are $n-1$ independent ghost fields $\left(c_{i} ; i=1, . ., n-1\right)$ in $C$. 


\section{Consistent anomalies.}

Now that the contact with the 2-dim Y-M theory has been made, the calculation of the consistent anomaly is easy. The Chern-Simons action,

$$
S=\int \operatorname{Tr}\left(\mathcal{A} d \mathcal{A}+\frac{2}{3} \mathcal{A} \mathcal{A} \mathcal{A}\right)
$$

which is defined on a three dimensional compact manifold, having $\Sigma$ as boundary, can be viewed as the integral of a Chern-Weil characteristic polynomial $T_{3}^{0}$ of rank 3. The lower index denotes the form degree and the upper index the ghost number. Consequently, by applying the BRST operator (17) to this polynomial, we obtain the descent equations

$$
\begin{gathered}
s T_{3}^{0}+d T_{2}^{1}=0, \\
s T_{2}^{1}+d T_{1}^{2}=0, \\
s T_{1}^{2}+d T_{0}^{3}=0, \\
s T_{0}^{3}=0,
\end{gathered}
$$

where the explicit expressions of the cocycles are given by

$$
\begin{aligned}
T_{2}^{1} & =3 \operatorname{Tr}\left(C \Omega \Omega^{*}-C \Omega^{*} \Omega\right) d z d \bar{z} \\
T_{1}^{2} & =3 \operatorname{Tr}\left(C^{2} \Omega d z+C^{2} \Omega^{*} d \bar{z}\right) \\
T_{0}^{3} & =\operatorname{Tr}\left(C^{3}\right)
\end{aligned}
$$

It is obvious that a cohomological analysis can determine the anomaly only modulo a (possibly vanishing) multiplicative constant whose value can be calculated, for a given model, through other methods, such as Feynman diagrams computation. Unless otherwise stated, what we call anomalies in this cohomological analysis have to be understood as possible candidates. They are non-trivial solutions of the Wess-Zumino consistency condition [14.

In fact the expressions above are valid only on the plane and on the sphere. Generalization to a Riemann surface of higher genus has been made only in the case of the bosonic string [15] and in the $W_{3}$ case [13] by introducing by hand a projective connection which is holomorphic and BRST inert. The present formalism allows us also to achieve a formulation of the anomaly associated to a given $W_{n}$-model which is well-defined on $\Sigma$. This formulation has the advantage of being self-contained, since the fields used to render the local expressions valid on any local coordinate chart on $\Sigma$ are the field $\tilde{\rho}_{n-2}$ which, under a conformal change of coordinates transforms as a projective connection, and the projective invariants which transform homogeneously, i.e are sections of $k^{n-i}$.

Instead of calculating the anomaly and its cocycles by using the Chern-Weil characteristic polynomial, it is also possible to start from the well-known 2-dim Y-M (non-integrated) anomaly $\operatorname{Tr}(C d \mathcal{A})$, resulting in 


$$
a_{2}^{1}=3 \operatorname{Tr}\left[C\left(\partial \Omega^{*}-\bar{\partial} \Omega\right)\right] d z d \bar{z} .
$$

This expression of the anomaly is shown to be equivalent to (22) thanks to the pure gauge condition (7). Moreover one can show that the 2nd term in the r.h.s of (25) is a well-defined density on $\Sigma$ so that the integration makes sense.

For later convenience let us consider (in fact as we have verified on the explicit examples $W_{2}$ and $W_{3}$ this choice is the most economical one)

$$
\sigma_{2}^{1}=3 \operatorname{Tr}\left(\Omega^{*} s \Omega-C \bar{\partial} \Omega\right) d z d \bar{z} .
$$

Indeed, using the transformation laws (4, 1, 11) and noting that by construction $\bar{\partial} \Lambda_{a b}=0$, we can easily verify that $\sigma_{2}^{1}$ is a well-defined 2 -form. This expression of the anomaly is related to the formula (22) given by the Y-M formalism by

$$
\sigma_{2}^{1}=T_{2}^{1}-3 \operatorname{Tr}\left(\bar{\partial}(C \Omega)-s\left(\Omega^{*} \Omega\right)\right) d z d \bar{z} .
$$

Then a well-defined system of descent equations is given by the following cocycles

$$
\begin{aligned}
& \sigma_{2}^{1}=3 \operatorname{Tr}\left(\Omega^{*} \partial C-C \partial \Omega^{*}+2 C\left(\Omega \Omega^{*}-\Omega^{*} \Omega\right)\right) d z d \bar{z} \\
& \sigma_{1}^{2}=-3 \operatorname{Tr}\left(C \partial C+2 C^{2} \Omega\right) d z+\operatorname{Tr}\left(\Omega^{*} C^{2}\right) d \bar{z} \\
& \sigma_{0}^{3}=\operatorname{Tr}\left(C^{3}\right)
\end{aligned}
$$

where the expression (28) is obtained by substituting in (26) $s \Omega$ and $\bar{\partial} \Omega$ by (14) and (7) respectively. Thus the formalism presented above provides us with a completely algorithmic procedure for calculating the consistent and conformally covariant anomalies associated to a given $W_{n}$-model and the cocycles related to these anomalies by the system of descent equations.

The covariant version of the differential operators $\partial^{n}$, the so-called Bol operators $L_{n}$ are well known [17]. Since the BRST transformations of the fields are also covariant quantities, these operators will appear naturally both in the formulation of these laws and in the expression of the covariant cocycles and anomaly. For later reference we display the explicit expressions of $L_{3}$ and $L_{5}$.

$$
\begin{aligned}
& L_{3}(R)=\partial^{3}+2 R \partial+(\partial R) \\
& L_{5}(R)=\partial^{5}+10 R \partial^{3}+15(\partial R) \partial^{2}+\left[9\left(\partial^{2} R\right)+16 R^{2}\right] \partial+2\left[\left(\partial^{3} R\right)+8 R(\partial R(R), 32)\right.
\end{aligned}
$$

Let us stress that the Bol operator $L_{n}$ is only covariant when acting on a conformal field of conformal dimension $\left(\frac{(1-n)}{2}\right)$ in the holomorphic sector, provided $R$ transforms with the Schwarzian derivative under a conformal change of coordinates: 


$$
R_{b}=k^{2}\left(R_{a}-S\left(z_{b}, z_{a}\right)\right)
$$

5. The induced gravity $W_{3}$.

We illustrate this construction for the $W_{3}$ algebra. First a technical problem appears for $n \geq 3$ : the geometrical fields parametrizing the matrix $W$ are not the physical fields since in general they do not transform homogeneously when one goes from one chart to another. Combinations $H$ of these fields which are sections of the fiber bundle $k^{p}, \bar{k} k^{-q}$ have to be considered; they transform as differentials under a conformal change of coordinates

$$
H_{b}=H_{a} k^{h} \bar{k}
$$

where $h=p$ or $-q$ is the conformal weight of the field $H$ ?

The sets of pairs of such fields used in the $n=3$ case are the generalized Beltrami coefficients $\left(\mu_{\bar{z}}^{z}, \mu_{\bar{z}}^{z z}\right)$, the projective connection $\rho_{z z}$ and the associated projective invariant $\rho_{z z z}$ and the ghosts $\left(c^{z}, c^{z z}\right)$. Now the components of the connection $\mathcal{A}$ are

$$
\begin{aligned}
& \Omega=\left(\begin{array}{ccc}
0 & 1 & 0 \\
0 & 0 & 1 \\
\rho_{z z z} & \rho_{z z} & 0
\end{array}\right) \\
& \Omega^{*}=\left(\begin{array}{ccc}
\frac{1}{6} \partial^{2} \mu_{\bar{z}}^{z z}-\frac{2}{3} \mu_{\bar{z}}^{z z} \rho_{z z}-\partial \mu_{\bar{z}}^{z} & \mu_{\bar{z}}^{z}-\frac{1}{2} \partial \mu_{\bar{z}}^{z z} & \mu_{\bar{z}}^{z z} \\
\partial \Omega_{00}^{*}-\frac{1}{2} \mu_{\bar{z}}^{z z} \partial \rho_{z z} & -\frac{1}{3}\left(\partial^{2} \mu_{\bar{z}}^{z z}-\mu_{\bar{z}}^{z z} \rho_{z z}\right) & \mu_{\bar{z}}^{z}+\frac{1}{2} \partial \mu_{\bar{z}}^{z z} \\
\partial \Omega_{01}^{*}+\partial\left(\mu_{\bar{z}}^{z z} \rho_{z z z}\right)+ & \frac{1}{2} \partial \Omega_{11}^{*}-\partial^{2} \mu_{\bar{z}}^{z}+\mu_{\bar{z}}^{z} \rho_{z z} & \partial \Omega_{12}^{*}+\Omega_{11}^{*} \\
\mu_{\bar{z}}^{z} \rho_{z z z}+\frac{1}{2} \mu_{\bar{z}}^{z} \partial \rho_{z z} & +\mu_{\bar{z}}^{z z} \rho_{z z z} &
\end{array}\right)
\end{aligned}
$$

whereas the ghost matrix is deduced from the $\Omega^{*}$ matrix by substituting for the generalized Beltrami coefficients $\mu_{\bar{z}}^{z}, \mu_{\bar{z}}^{z z}$, the ghosts $c^{z}$ and $c^{z z}$ respectively.

$$
C=\left(\begin{array}{ccc}
\frac{1}{6} \partial^{2} c^{z z}-\frac{2}{3} c^{z z} \rho_{z z}-\partial c^{z} & c^{z}-\frac{1}{2} \partial c^{z z} & c^{z z} \\
\partial C_{00}-\frac{1}{2} c^{z z} \partial \rho_{z z} & -\frac{1}{3}\left(\partial^{2} c^{z z}-c^{z z} \rho_{z z}\right) & c^{z}+\frac{1}{2} \partial c^{z z} \\
\partial C_{01}+\partial\left(c^{z z} \rho_{z z z}\right)+ & \frac{1}{2} \partial C_{11}-\partial^{2} c^{z}+c^{z} \rho_{z z} & \partial C_{12}+C_{11} \\
+c^{z} \rho_{z z z}+\frac{1}{2} c^{z} \partial \rho_{z z} & +c^{z z} \rho_{z z z} &
\end{array}\right)
$$

\footnotetext{
${ }^{2}$ In sect.2 these fields were denoted by a tilde superscript which is omitted here to simplify the notations.
} 
Projecting down the matrix equations $(13,14)$ to the components $(0,1)$ and $(0,2)$ for instance, and (15) to $(2,0)$ and $(2,1)$ we obtain the automatically nilpotent laws

$$
\begin{aligned}
s c^{z z} & =2 c^{z z} \partial c^{z}-\partial c^{z z} c^{z} \\
s c^{z} & =c^{z} \partial c^{z}-\frac{1}{6} c^{z z} \partial^{3} c^{z z}+\frac{1}{4} \partial c^{z z} \partial^{2} c^{z z}-\frac{2}{3} \partial c^{z z} c^{z z} \rho_{z z}, \\
s \mu_{\bar{z}}^{z z} & =\overline{\mathcal{D}}_{-2}\left(\mu_{\bar{z}}^{z}\right) c^{z z}+c^{z} \partial \mu_{\bar{z}}^{z z}-2 \mu_{\bar{z}}^{z z} \partial c^{z}, \\
s \mu_{\bar{z}}^{z} & =\overline{\mathcal{D}}_{-1}\left(\mu_{\bar{z}}^{z}\right) c^{z}-\frac{1}{12}\left(2 c^{z z} \partial^{3}-3 \partial c^{z z} \partial^{2}+3 \partial^{2} c^{z z} \partial-2 \partial^{3} c^{z z}\right) \mu_{\bar{z}}^{z z} \\
& +\frac{2}{3}\left(c^{z z} \partial \mu_{\bar{z}}^{z z}-\mu_{\bar{z}}^{z z} \partial c^{z z}\right) \rho_{z z}, \\
s \rho_{z z}= & -2 L_{3}\left(-\frac{1}{2} \rho_{z z}\right) c^{z}+\left(2 c^{z z} \partial \rho_{z z z}+3 \partial c^{z z} \rho_{z z z}\right), \\
s \rho_{z z z} & =\frac{1}{6} L_{5}\left(-\frac{1}{2} \rho_{z z}\right) c^{z z}+3 \rho_{z z z} \partial c^{z}+c^{z} \partial \rho_{z z z},
\end{aligned}
$$

where we have introduced the following derivative acting on any field of conformal dimension $J$ as:

$$
\overline{\mathcal{D}}_{J}\left(\mu_{\bar{z}}^{z}\right)=\bar{\partial}-\mu_{\bar{z}}^{z} \partial-J \partial \mu_{\bar{z}}^{z}
$$

These laws have to be complemented by the constraints of the holomorphic conditions

$$
\begin{gathered}
\bar{\partial} \rho_{z z}=-2 \quad L_{3}\left(-\frac{1}{2} \rho_{z z}\right) \mu_{\bar{z}}^{z}+\left(2 \mu_{\bar{z}}^{z z} \partial \rho_{z z z}+3 \partial \mu_{\bar{z}}^{z z} \rho_{z z z}\right), \\
\bar{\partial} \rho_{z z z}=\frac{1}{6} L_{5}\left(-\frac{1}{2} \rho_{z z}\right) \mu_{\bar{z}}^{z z}+3 \rho_{z z z} \partial \mu_{\bar{z}}^{z}+\mu_{\bar{z}}^{z} \partial \rho_{z z z}
\end{gathered}
$$

which express, in the parametrization (35, 36) of $\Omega$ and $\Omega^{*}$, the flatness of the connection. One should notice that the BRST transformation of the $\rho$ fields can be obtained from $(45,46)$ through the replacement of $\bar{\partial}$ by the operator $s$ and of $\left(\mu_{\bar{z}}^{z}, \mu_{\bar{z}}^{z z}\right)$ by $\left(c^{z}, c^{z z}\right)$, as discussed at the end of Sect.3.

The covariant non-integrated $W_{3}$ anomaly is now obtained by replacing (35, 36, 37) in (28). Modulo total derivatives, it reads

$$
\begin{aligned}
\sigma_{2}^{1} & =\left[\frac{1}{6}\left(\mu_{\bar{z}}^{z z} L_{5}\left(-\frac{1}{2} \rho_{z z}\right) c^{z z}-c^{z z} L_{5}\left(-\frac{1}{2} \rho_{z z}\right) \mu_{\bar{z}}^{z z}\right)-2\left(\mu_{\bar{z}}^{z} L_{3}\left(-\frac{1}{2} \rho_{z z}\right) c^{z}\right.\right. \\
& \left.\left.-c^{z} L_{3}\left(-\frac{1}{2} \rho_{z z}\right) \mu_{\bar{z}}^{z}\right)+2 \rho_{z z z}\left(\partial c^{z z} \mu_{\bar{z}}^{z}-2 \partial \mu_{\bar{z}}^{z} c^{z z}+2 \partial c^{z} \mu_{\bar{z}}^{z z}-c^{z} \partial \mu_{\bar{z}}^{z z}\right)\right] d z d(\bar{E} 47)
\end{aligned}
$$

Using (45, 46) the covariant anomaly (47) takes the compact form (compare with $(26))$

$$
\sigma=\mu s \rho-c \bar{\partial} \rho
$$


thus proving the overall consistency of our results 5 .

A different form of the $W_{3}$ anomaly has been found by the authors of [4], as solution of the Wess-Zumino consistency conditions. It is easy to show that the BRST operator corresponding to their transformation algebra coincides with that given by eqs.(38 43). Their expression for the anomaly, rewritten in terms of our fields reads

$$
\tilde{\sigma}_{2}^{1}=\left\{-\frac{1}{3} c^{z z}\left(\partial^{5} \mu_{\bar{z}}^{z z}-4\left(\rho_{z z}^{2} \partial \mu_{\bar{z}}^{z z}+\rho_{z z} \partial \rho_{z z} \mu_{\bar{z}}^{z z}\right)\right)+4 c^{z} \partial^{3} \mu_{\bar{z}}^{z}\right\} d z d \bar{z}
$$

We have explicitly checked that both expressions (47) and (48) are in fact cocycles of the BRST algebra ( once the holomorphy conditions are taken into account). Accordingly they have to be equivalent, and, in fact, the following relation holds (again modulo the holomorphy conditions)

$$
\sigma_{z \bar{z}}^{1}+\tilde{\sigma}_{z \bar{z}}^{1}=\bar{\partial} \Delta_{z}^{1}+\partial \Delta_{\bar{z}}^{1}-s \Delta_{z \bar{z}}^{0}
$$

Since this equation is invariant under the transformations

$$
\Delta_{z \bar{z}}^{0} \rightarrow \Delta_{z \bar{z}}^{0}+\partial u_{\bar{z}}-\bar{\partial} u_{z} ; \quad \Delta_{z}^{1} \rightarrow \Delta_{z}^{1}+\partial v-s u_{z} ; \quad \Delta_{\bar{z}}^{1} \rightarrow \Delta_{\bar{z}}^{1}+\bar{\partial} v-s u_{\bar{z}} ;
$$

the general form of the $\Delta$ 's is given by

$$
\begin{aligned}
& \Delta_{z \bar{z}}^{0}=-2 \mu_{\bar{z}}^{z z} \rho_{z z z}-2 \mu_{\bar{z}}^{z} \rho_{z z}+\partial u_{\bar{z}}-\bar{\partial} u_{z} \\
& \Delta_{z}^{1}=-2 c^{z z} \rho_{z z z}-2 c^{z} \rho_{z z}+\partial v-s u_{z} \\
& \Delta_{\bar{z}}^{1}=4\left(c^{z} \partial^{2} \mu_{\bar{z}}^{z}-\partial c^{z} \partial \mu_{\bar{z}}^{z}+\partial^{2} c^{z} \mu_{\bar{z}}^{z}\right)- \\
& \frac{1}{3}\left(\partial^{4} c^{z z} \mu_{\bar{z}}^{z z}-\partial^{3} c^{z z} \partial \mu_{\bar{z}}^{z z}+\partial^{2} c^{z z} \partial^{2} \mu_{\bar{z}}^{z z}-\partial c^{z z} \partial^{3} \mu_{\bar{z}}^{z z}+c^{z z} \partial^{4} \mu_{\bar{z}}^{z z}\right)+ \\
& \frac{1}{3}\left(4 \partial^{2} c^{z z} \mu_{\bar{z}}^{z z}-\frac{5}{2} \partial c^{z z} \partial \mu_{\bar{z}}^{z z}+c^{z z} \partial^{2} \mu_{\bar{z}}^{z z}\right) \rho_{z z}-\frac{1}{3}\left(c^{z z} \partial \mu_{\bar{z}}^{z z}-\frac{7}{2} \partial c^{z z} \mu_{\bar{z}}^{z z}\right) \partial \rho_{z z}+ \\
& \frac{1}{3} c^{z z} \mu_{\bar{z}}^{z z} \partial^{2} \rho_{z z}-2 c^{z} \mu_{\bar{z}}^{z} \rho_{z z}+2\left(c^{z} \mu_{\bar{z}}^{z z}-c^{z z} \mu_{\bar{z}}^{z}\right) \rho_{z z z}+\bar{\partial} v-s u_{\bar{z}}
\end{aligned}
$$

where $u_{z}, u_{\bar{z}}$, are the holomorphic, antiholomorphic components of an arbitrary 1-form with ghost number 0 ( the inspection of possible terms fixes $u_{z}=0$ and $u_{\bar{z}}$ to be an arbitrary linear combination of the monomials $\partial \mu_{\bar{z}}^{z}, \partial^{2} \mu_{\bar{z}}^{z z}$ and $\mu_{\bar{z}}^{z z} \rho_{z z}$ ) and $v$ is an an arbitrary 0-form with ghost number 1 (so an arbitrary linear combination of $\partial c^{z}, \partial^{2} c^{z z}$ and $c^{z z} \rho_{z z}$ ). Evidently, the relation between the (integrated) anomalies is independent of the choice of $u_{\bar{z}}$ and $v$ since we can

\footnotetext{
${ }^{3} \mathrm{~A}$ similar expression for $\sigma_{2}^{1}$ has been obtained in ref. 16 .
} 
disregard $\Delta_{z}^{1}, \Delta_{\bar{z}}^{1}$ and the term involving $u_{\bar{z}}$ in $\Delta_{z \bar{z}}^{0}$ because arising from total derivatives.

It should be noticed that both expressions (47) and (48) contain, besides the leading terms $\mu_{\bar{z}}^{z} \partial^{3} c^{z}$ and $\mu_{\bar{z}}^{z z} \partial^{5} c^{z z}$, additional contributions which are necessary to insure the consistency of the anomaly.

Leading terms of the form $\mu_{\bar{z}}^{z \ldots z} \partial^{2 n-1} c^{z \ldots z}$ (called universal anomalies by Hull [18, 2]) are present in all models obtained by coupling a system of free scalar fields to $W_{n}$ gravity [18, 19. Evidently, conformal invariance requires the occurrence of additional terms that together with Hull's universal terms lead to the necessary Bol operators. Thus, these terms are easily obtained. On the other side, as discussed above, additional terms, involving the higher spin $\rho$ fields, are also needed, which cannot be easily guessed. However, in our approach the general expressions for $C, \Omega$ and $\Omega^{*}$ given in [3] and eq. (26) allow us to obtain straightforwardly them by a mere repetition of the computations.

We would like to thank F.Biet, Y.Noirot and R.Zucchini for valuable discussions.

Note added: after submition of the present paper, it appeared a work where is discussed a comparison between the anomalies of ref. [16] and ref. [4, see P. Watts, Generalized Wess-Zumino consistency conditions for pure $\mathcal{W}_{3}$ anomalies. CPT-95/P.3237; hep-th/9509044.

\section{References}

[1] P.Bouwknegt and K.Schoutens, Phys. Rep. 223 (1993) 183

[2] C.M.Hull, Lectures on W-Gravity, W-Geometry and W-Strings, Trieste Summer School in High Energy Physics and Cosmology (1992)

[3] R. Zucchini, Class. Quantum. Gravity 10 (1993) 253

[4] H. Ooguri, K. Schoutens, A. Sevrin and P. van Nieuwenhuizen, Commun. Math.Phys. 145 (1992) 515

[5] P. Di Francesco, C. Itzykson and J.-B. Zuber, Commun. Math. Phys. 140 (1991) 543

[6] M. Bauer, P. Di Francesco, C. Itzykson and J.-B. Zuber, Nucl. Phys. B362 (1991) 515

[7] A.M.Polyakov, Mod. Phys. Lett. A2 (1987) 893

\footnotetext{
${ }^{4}$ The anomalous Ward identities in the $W_{n}$ case have been already discussed in refs. 110, 11.
} 
[8] K.Yoshida, Int. J. Mod. Phys. A7 (1992) 4353

[9] R.Zucchini, Commun. Math. Phys. 152 (1993) 269

[10] A. Bilal, V.V. Fock and I.I.Kogan, Nucl. Phys. B359 (1991) 635

[11] A.Das, W.J.Huang and S.Roy, Int. J. Mod. Phys. A7 (1992) 3447

[12] C.Becchi, Nucl. Phys. B304 (1988) 513

[13] J.de Boer and J.Goeree, Nucl. Phys. B401 (1993) 369

[14] W.Bardeen and B.Zumino, Nucl. Phys. B244 (1984) 421

[15] S. Lazzarini and R. Stora, in 'Knots, topology and quantum field theories', Proc. 13th Johns Hopkins Workshop, eds Lusanna (World Scientific, Singapore, 1989)

[16] D. Garajeu, R. Grimm and S. Lazzarini, $\mathcal{W}$-Gauge structures and their anomalies.CPT-94/P.3078; hep-th/9411125

[17] F. Gieres, Int. J. Mod. Phys. A8 (1993) 1

[18] C.M.Hull, Nucl.Phys. B367 (1991) 731

[19] K. Schoutens, A. Sevrin and P. van Nieuwenhuizen, Nucl.Phys. B364 (1991) 584 